\title{
A kinetic model of reactive crystal surfaces
}

Cite as: AIP Conference Proceedings 2132, 130003 (2019); https://doi.org/10.1063/1.5119623

Published Online: 05 August 2019

Kazuo Aoki, and Vincent Giovangigli

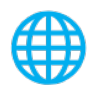

Export citation

\section{Conference Proceedings}

Get $30 \%$ off all print proceedings!

\section{Enter Promotion Code PDF30 at checkout}

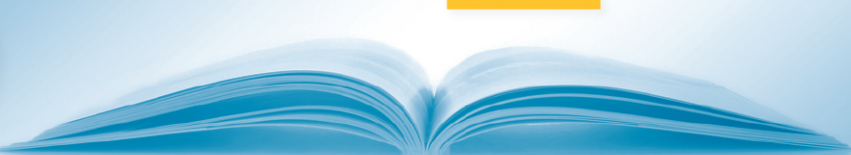




\title{
A Kinetic Model of Reactive Crystal Surfaces
}

\author{
Kazuo Aoki ${ }^{\dagger a)}$ and Vincent Giovangigli $\left.{ }^{\star b}\right)$ \\ ${ }^{\dagger}$ National Center for Theoretical Sciences, National Taiwan University, Taipei 10617, TAIWAN and \\ Department of Mathematics, National Cheng Kung University, Tainan 70101, TAIWAN \\ ${ }^{\star}$ CMAP-CNRS, École Polytechnique, 91128 Palaiseau, FRANCE \\ a)kazuo.aoki.22v@st.kyoto-u.ac.jp \\ b) vincent.giovangigli@ polytechnique.fr
}

\begin{abstract}
A kinetic model describing chemical reactions on a crystal surface is introduced. The Boltzmann equations involve particles interacting with potentials generated by fixed crystal particles and interacting with a phonon gas describing the fluctuating part of the potentials. Chemical reactions between gas/physisorbed, chemisorbed and crystal species are taken into account. The phonons are assumed to be at equilibrium for the sake of simplicity. A modified kinetic entropy is introduced for the coupled system and the $\mathrm{H}$ theorem is established. Using a fluid scaling and the Chapman-Enskog asymptotic method, species fluid boundary conditions involving heterogeneous reactions are recovered at the surface.
\end{abstract}

\section{INTRODUCTION}

Chemical reactions on surfaces are notably involved during hypersonic reentry $[1,2,3]$, combustion $[4,5]$, condensation and evaporation [6,7], and chemical deposition reactors [8]. We investigate in this work a kinetic model for heterogeneous chemistry—at a scale intermediate between molecular simulation [9] and fluid models [10]—and derive rigorously species fluid boundary conditions using the Chapman-Enskog method.

Detailed kinetic models of gas crystal interaction involve Boltzmann equations taking into account the interaction of gas particles with an average surface potential as well as phonons near the surface boundary $[11,12,13,14,15$, $16,17]$. These models have been extended to include chemisorption in previous work on adsorption of a single species [18] and are extended here to include surface chemistry. The species involved in the surface reactions are the physisorbed/gas particles, the chemisorbed particles, the crystal surface free sites $\mathrm{C}(\mathrm{s})$ and crystal particles bonded to chemisorbed species $\mathcal{C}(\mathrm{b})$. The crystal is supposed to be monatomic for the sake of simplicity and the chemisorbed particle may also move on the surface. The phonons are assumed to be at equilibrium for the sake of simplicity [12], although a kinetic equation for phonons may also be considered [11, 19, 20]. Using the atomic site formalism of heterogeneous chemistry, the chemical reactions are written in the form

$$
\sum_{i \in \mathcal{F}^{r}} \mathfrak{M}_{i} \rightleftarrows \sum_{k \in \mathcal{B}^{r}} \mathfrak{M}_{k}, \quad r \in \mathfrak{R},
$$

where $\mathfrak{M}_{i}$ is the symbol of the $i$ th species that may either be a gas/physisorbed particle, a chemisorbed particle, or a crystal particle $\mathcal{C}(\mathrm{s})$ or $\mathcal{C}(\mathrm{b})$. The sets $\mathcal{F}^{r}$ and $\mathcal{B}^{r}$ denotes respectively the indices of reactants and products and $\Re$ the indices for chemical reactions. The surface chemical reaction mechanism is thus arbitrarily complex and cover Langmuir-Hinshelwood as well as Eley-Rideal reaction types. A modified kinetic entropy is introduced for the coupled system and the $\mathrm{H}$ theorem is established.

A Chapman-Enskog type expansion is then investigated with a fluid scaling of the kinetic equations. A multiscale asymptotic analysis is performed and the fluid Stefan velocity associated with total mass production at the surface is naturally assumed to be small. The inner structure of the physisorbate, the chemisorbate, and the crystal surface layers are analyzed at zeroth order and closely related to interaction potentials. The gas species diffusive fluxes and the Stefan gas flow then results from the physisorbed/gas particle production by surface chemistry. The traditional species fluid boundary conditions are thus recovered and involve the surface chemistry production rates [10].

31st International Symposium on Rarefied Gas Dynamics

AIP Conf. Proc. 2132, 130003-1-130003-8; https://doi.org/10.1063/1.5119623

Published by AIP Publishing. 978-0-7354-1874-5/\$30.00 


\section{KINETIC MODEL}

\section{Kinetic equations}

We consider a gas mixture composed by monatomic or polyatomic species indexed by $i \in S_{\mathrm{g}}$ near a crystal surface. These particules may approach the surface and be physisorbed but may also be chemisorbed by forming chemical bonds with the crystal particles. The chemisorbed species - that are different chemical species—are indexed by $i \in S_{\mathrm{c}}$. We also consider the crystal particles on the surface that are free sites $\mathcal{C}(\mathrm{s})$ or crystal particles bonded to chemisorbed particles $\mathrm{C}(\mathrm{b})$. The resulting species indexing set $S$ for heterogeneous chemistry then reads $S=S_{\mathrm{g}} \cup S_{\mathrm{c}} \cup\{s, b\}$. The Boltzmann equations governing the species are in the form $[11,12,13,24,18]$

$$
\partial_{t} f_{i}+\boldsymbol{c}_{i} \cdot \boldsymbol{\partial}_{\boldsymbol{x}} f_{i}-\frac{1}{m_{i}} \boldsymbol{\partial}_{\boldsymbol{x}} \mathrm{w}_{i} \cdot \boldsymbol{\partial}_{\boldsymbol{c}_{i}} f_{i}=\sum_{j \in S_{\mathrm{g}}} J_{i j}\left(f_{i}, f_{j}\right)+J_{i, \mathrm{ph}}\left(f_{i}\right)+C_{i}, \quad i \in S,
$$

where $\partial_{t}$ denotes the time derivative operator, $\boldsymbol{\partial}_{\boldsymbol{x}}$ the space derivative operator, $i \in S$ the species index, $f_{i}\left(t, \boldsymbol{x}, \boldsymbol{c}_{i}\right)$ the $i$ th species distribution function, $\boldsymbol{c}_{i}$ the $i$ th species particle velocity, $m_{i}$ the particle mass, $\mathrm{w}_{i}$ the interaction potential between fixed crystal particles and the particles of the $i$ th species (between fixed interior crystal particles and surface crystal particles when $i \in\{\mathrm{s}, \mathrm{b}\}), \partial_{\boldsymbol{c}_{i}}$ the velocity derivative operator, $J_{i j}$ the collision operator between the $i$ th and the $j$ th gas/physisorbed species, $J_{i, \mathrm{ph}}$ the particle-phonon collision operator, and $C_{i}$ the chemistry source term. The solid surface is assumed to be planar and located at $z=0$ with the spatial coordinates written $\boldsymbol{x}=(x, y, z)^{t}=\left(\boldsymbol{x}_{\|}, z\right)^{t}$ and $\boldsymbol{e}_{z}$ denotes the base vector in the normal direction oriented towards the gas. The particle collision operator $J_{i j}$ are in the traditional form [22,23] and only collisions between gas/physisorbed species $i, j \in S_{\mathrm{g}}$, are taken into account. The collisional invariants of the particle collision operator $J_{i j}, i, j \in S$, are classically associated with particle number $\left(\delta_{i l}\right)_{i \in S_{\mathrm{g}}}, l \in S_{\mathrm{g}}$, where $\delta_{i l}$ is the Kronecker symbol, momentum $\left(m_{i} c_{i v}\right)_{i \in S_{\mathrm{g}}}, v \in\{1,2,3\}$, as well as total energy $\left(\frac{1}{2} m_{i}\left|\boldsymbol{c}_{i}\right|^{2}+\mathrm{E}_{i \mathrm{i}}+\mathrm{w}_{i}\right)_{i \in S_{\mathrm{g}}}$. For gas/physisorbed species, the collision operators $J_{i, \mathrm{ph}}$ and $C_{i}$ both vanish far from the surface as well as the potential $\mathrm{w}_{i}$ in such a way that letting $z \rightarrow \infty$ in equation (2) yields the kinetic equation of the $i$ th species in the gas phase governing the gas distribution function $f_{\mathrm{g} i}\left(t, \boldsymbol{x}, \boldsymbol{c}_{i}\right)$ of the $i$ th species with $i \in S_{\mathrm{g}}$.

The interaction potentials summed over fixed crystal particles $\mathrm{w}_{i}$, are assumed to only depend on the normal coordinate $z$ for the sake of simplicity. These potentials are written in the form $\mathrm{w}_{i}=\mathrm{w}_{i}(z / \delta)$, where $\delta$ is a characteristic range of the surface potential also characteristic of the range of gas-phonons interaction and $\zeta=z / \delta$ is the corresponding rescaled normal coordinate. The potentials are such that $\lim _{\zeta \rightarrow 0} \mathrm{w}_{i}(\zeta)=+\infty$ and $\lim _{\zeta \rightarrow+\infty} \mathrm{w}_{i}(\zeta)=0$ for $i \in S_{\mathrm{g}}$, and $\lim _{\zeta \rightarrow \zeta_{i}^{-}} \mathrm{w}_{i}(\zeta)=+\infty$ and $\lim _{\zeta \rightarrow \zeta_{i}^{+}} \mathrm{w}_{i}(\zeta)=+\infty$ for $i \in S_{\mathrm{c}} \cup\{\mathrm{s}, \mathrm{b}\}$, so that chemisorbed and crystal species are localized over $\left(\zeta_{i}^{-}, \zeta_{i}^{+}\right)$with $i \in S_{\mathrm{c}} \cup\{\mathrm{s}, \mathrm{b}\}$. These interaction potentials usually involve an attractive zone and a repulsing zone as Lennard-Jones potentials integrated over all crystal particles. Periodic potential variations parallel to the surface are not taken into account as well as lateral interactions between chemisorbed species. The surface potential $\mathrm{w}_{i}, i \in S$, are also assumed to be independent of the coverage $\theta$ for the sake of simplicity. These simplifications are notably feasible when the chemisorbed species have relatively small masses and the coverage of the surface $\theta$ is low.

\section{Phonon collision operators}

The species distribution functions at physical equilibrium are given by $f_{i}^{\mathrm{e}}=n_{i} \mathrm{M}_{i}$ where $n_{i}$ denotes the number of particle per unit volume, $\mathrm{M}_{i}$ the wall Maxwellian of the $i$ th species and the superscript ${ }^{\mathrm{e}}$ stands for physical equilibrium. The wall Maxwellian distribution is in the form

$$
\mathrm{M}_{i}=\left(\frac{m_{i}}{2 \pi k_{\mathrm{B}} T_{\mathrm{w}}}\right)^{3 / 2} \frac{a_{i \mathrm{I}}}{\mathrm{z}_{i}^{\text {int }}} \exp \left(-\frac{m_{i}\left|\boldsymbol{c}_{i}\right|^{2}}{2 k_{\mathrm{B}} T_{\mathrm{w}}}-\frac{\mathrm{E}_{i \mathrm{I}}}{k_{\mathrm{B}} T_{\mathrm{w}}}\right), \quad \mathrm{z}_{i}^{\text {int }}=\sum_{\mathrm{I} \in Q_{i}} a_{i \mathrm{I}} \exp \left(-\frac{\mathrm{E}_{i \mathrm{I}}}{k_{\mathrm{B}} T_{\mathrm{w}}}\right),
$$

where $m_{i}$ denotes the mass of the $i$ th species, $k_{\mathrm{B}}$ the Boltzmann constant, $T_{\mathrm{w}}$ the wall temperature, $\mathrm{I}$ the quantum state, $a_{i \mathrm{II}}$ the degeneracy of the ith state, $Q_{i}$ the indexing set for the quantum states of the $i$ th species, $\mathrm{z}_{i}^{\text {int }}$ the internal partition function of the $i$ th species, and $\mathrm{E}_{i \mathrm{I}}$ the energy of the $i$ th species in the ith state. We introduce for future use the modified Maxwellian distributions $\mathrm{m}_{i}=\mathrm{M}_{i} \exp \left(-\mathrm{w}_{i} / k_{\mathrm{B}} T_{\mathrm{w}}\right)$ for $i \in S$ that will play a key role in the analysis of the adsorbate layer. With the inclusion of the interaction potential $\mathrm{w}_{i}$, the distribution $\mathrm{m}_{i}$ is indeed such that $\partial_{t} \mathrm{~m}_{i}=0, J_{i, \mathrm{ph}}\left(\mathrm{m}_{i}\right)=0$, $\partial_{\| 1} \mathrm{~m}_{i}=0, c_{i z} \partial_{z} \mathrm{~m}_{i}-\frac{1}{m_{i}} \partial_{z} \mathrm{w}_{i} \partial_{c_{i z}} \mathrm{~m}_{i}=0$. The modified Maxwellian distributions $\mathrm{m}_{i}, i \in S$, thus appear as natural solutions of thin layer kinetic equations in a potential field with phonon interactions. In particular, the unperturbed surface layer distribution $f_{\sigma}^{\mathrm{e}}$ is in the form $f_{\sigma}^{\mathrm{e}}=n_{\sigma} \mathrm{M}_{\sigma}$ where $n_{\sigma}$ denotes the number density of the surface layer with 
$n_{\sigma}=\bar{n}_{\sigma} \exp \left(-\frac{\mathrm{w}_{\sigma}}{k_{\mathrm{B}} T_{\mathrm{w}}}\right), \mathrm{w}_{\sigma}=\mathrm{w}_{\mathrm{s}}=\mathrm{w}_{\mathrm{b}}$ is the surface crystal layer potential, and $\bar{n}_{\sigma}$ is a constant characteristic of the crystal and its orientation.

The collision operators $J_{i, \mathrm{ph}}$ between phonons and particles are in the general form [11, 19, 20]

$$
J_{i, \mathrm{ph}}=\sum_{\mathrm{I}^{\prime} \in Q_{i}} \int\left(\left(f_{\mathrm{ph}}(\boldsymbol{q})+1\right) f_{i}\left(\boldsymbol{c}_{i}^{\prime}, \mathrm{I}^{\prime}\right) \frac{a_{i \mathrm{i}}}{a_{i I^{\prime}}}-f_{\mathrm{ph}}(\boldsymbol{q}) f_{i}\left(\boldsymbol{c}_{i}, \mathrm{I}\right)\right) \overline{\mathrm{W}}_{i, \mathrm{ph}} \mathrm{d} \boldsymbol{c}_{i}^{\prime} \mathrm{d} \boldsymbol{q}, \quad i \in S,
$$

where $f_{\mathrm{ph}}(\boldsymbol{q})$ denotes the phonon distribution function, $\boldsymbol{q}$ the phonon wave vector or quasi-momentum, $\boldsymbol{c}_{i}$ and $\boldsymbol{c}_{i}^{\prime}$ the particle velocities before and after the interaction, I and $\mathrm{I}^{\prime}$ the quantum states before and after the interaction, and $\overline{\mathrm{W}}_{i, \mathrm{ph}}$ a degeneracy averaged transition probability. The dilute approximation has been used for simplifying (4) and the appearing of the additional factor 1 in the gain term is a typical quantum effect $[11,19,20]$. The operator (4) corresponds to collisions such that $m_{i} \boldsymbol{c}_{i}=m_{i} \boldsymbol{c}_{i}^{\prime}+\boldsymbol{q}+\boldsymbol{b}$ where $\boldsymbol{b}$ is a vector of the reciprocal crystal lattice and there is another operator associated with collisions such that $m_{i} \boldsymbol{c}_{i}+\boldsymbol{q}=m_{i} \boldsymbol{c}_{i}^{\prime}+\boldsymbol{b}$ that leads to the same type of simplified source term $J_{i, \mathrm{ph}}$ and the details are omitted. The equilibrium relation corresponding to (4) reads $\left(f_{\mathrm{ph}}^{\mathrm{e}}(\boldsymbol{q})+1\right) f_{i}^{\mathrm{e}}\left(\boldsymbol{c}_{i}^{\prime}, \mathrm{I}^{\prime}\right) a_{i \mathrm{I}} / a_{i I^{\prime}}=$ $f_{\mathrm{ph}}^{\mathrm{e}}(\boldsymbol{q}) f_{i}^{\mathrm{e}}\left(\boldsymbol{c}_{i}, \mathrm{I}\right)$ where the superscript ${ }^{\mathrm{e}}$ stands for physical equilibrium and may be rewritten $\left(f_{\mathrm{ph}}^{\mathrm{e}}(\boldsymbol{q})+1\right) \mathrm{m}_{i}\left(\boldsymbol{c}_{i}^{\prime}, \mathrm{I}^{\prime}\right) a_{i \mathrm{il}} / a_{i i^{\prime}}=$ $f_{\mathrm{ph}}^{\mathrm{e}}(\boldsymbol{q}) \mathrm{m}_{i}\left(\boldsymbol{c}_{i}, \mathrm{I}\right)$. Dividing then the integrands in the collision term (4) by the later factor $f_{\mathrm{ph}}^{\mathrm{e}}(\boldsymbol{q}) \mathrm{m}_{i}\left(\boldsymbol{c}_{i}, \mathrm{I}\right)$ and further assuming that phonons are at equilibrium $f_{\mathrm{ph}}^{\mathrm{e}}=f_{\mathrm{ph}}$, it is obtained that

$$
J_{i, \mathrm{ph}}\left(f_{i}\right)=\sum_{\mathrm{I}^{\prime} \in Q_{i}} \int\left(\frac{f_{i}\left(\boldsymbol{c}_{i}^{\prime}, \mathrm{I}^{\prime}\right)}{\mathrm{m}_{i}\left(\boldsymbol{c}_{i}^{\prime}, \mathrm{I}^{\prime}\right)}-\frac{f_{i}\left(\boldsymbol{c}_{i}, \mathrm{I}\right)}{\mathrm{m}_{i}\left(\boldsymbol{c}_{i}, \mathrm{I}\right)}\right) \mathrm{W}_{i, \mathrm{ph}} \mathrm{d} \boldsymbol{c}_{i}^{\prime}, \quad i \in S,
$$

where $\mathrm{W}_{i, \mathrm{ph}}=\mathrm{m}_{i}\left(\boldsymbol{c}_{i}, \mathrm{I}\right) \int f_{\mathrm{ph}}^{\mathrm{e}}(\boldsymbol{q}) \overline{\mathrm{W}}_{i, \mathrm{ph}} \mathrm{d} \boldsymbol{q}$ denotes the resulting transition probability that satisfies the reciprocity relation $\mathrm{W}_{i, \mathrm{ph}}\left(\boldsymbol{c}_{i}, \mathrm{I}, \boldsymbol{c}_{i}^{\prime}, \mathrm{I}^{\prime}\right)=\mathrm{W}_{i, \mathrm{ph}}\left(\boldsymbol{c}_{i}^{\prime}, \mathrm{I}^{\prime}, \boldsymbol{c}_{i}, \mathrm{I}\right)$ and is nonzero only in the neighborhood of the surface [11, 12]. Contrarily to particle-particle collision operators, momentum and energy are not conserved by the collision operators $J_{i, \mathrm{ph}}$ since they may be given to phonons that are assumed to be at equilibrium and only the number of particles is conserved.

\section{Reactive collision operators}

The $r$ th surface chemical reaction has been written in the form (1) where $\mathcal{F}^{r}$ and $\mathcal{B}^{r}$ denote the indices for the reactants and products counted with their order of multiplicity and $\mathfrak{R}$ is the set of reaction indices. With the atomic site formalism, all particles involved in reactive collisions are properly taken into account in the symbolic description of the chemical reaction (1). The reactive collision term for the $i$ th species may be written $C_{i}(f)=\sum_{r \in \Re} C_{i}^{r}(f)$ where $C_{i}^{r}(f)$ represents the contribution of the $r$ th reaction [22, 23]. We denote by $v_{i r}^{\mathrm{f}}$ and $v_{i r}^{\mathrm{b}}$ the stoichiometric coefficients of the $i$ th species in the $r$ th reaction, that is, the multiplicity of species $i$ in $\mathcal{F}^{r}$ and $\mathcal{B}^{r}$, respectively, and by $\mathrm{F}^{r}$ and ${ }^{r}$ the indices of the quantum energy states of the reactants and products, respectively. We also denote by $\mathcal{F}_{i}^{r}$ the subset of $\mathcal{F}^{r}$ where the index $i$ has been removed once with similar notation for $\mathcal{B}_{k}^{r}, \mathrm{~F}_{\mathrm{I}}^{r}$ and $\mathrm{B}_{\mathrm{K}}^{r}$. The reactive collision term $C_{i}^{r}(f)$ associated with (1) may be written $[22,23]$

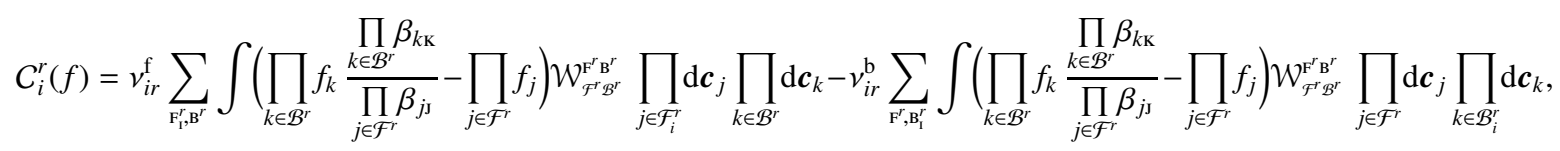

where $\beta_{i \mathrm{I}}=\mathrm{h}_{\mathrm{p}}^{3} /\left(a_{i \mathrm{I}} m_{i}^{3}\right), \mathrm{h}_{\mathrm{P}}$ is the Planck constant, and $\mathcal{W}_{\mathcal{F}^{\prime} B^{r}}^{\mathrm{F}^{r}{ }^{r}}$ denotes the transition probability that a collision between the reactants $\mathcal{F}^{r}$ with energies $\mathrm{F}^{r}$ leads to the products $\mathcal{B}^{r}$ with energies $\mathrm{B}^{r}$. The summation over $\mathrm{F}^{r}$ represents the sum over all quantum indices $\mathrm{J}$ for all $j \in \mathcal{F}^{r}$ with similar conventions for $\mathrm{F}_{1}^{r}, \mathrm{~B}^{r}$, and $\mathrm{B}_{1}^{r}$. The following reciprocity relations hold between transition probabilities $[22,23] \mathcal{W}_{\mathcal{F}^{r} g^{r}}^{\mathrm{F}^{r} r^{r}} \prod_{k \in \mathcal{B}^{r}} \beta_{k \mathrm{~K}}=\mathcal{W}_{\mathcal{G}^{r} \mathcal{F}^{r}}^{\mathrm{B}^{r} \mathrm{r}^{r}} \prod_{j \in \mathcal{F}} \beta_{j \mathrm{j}}$. It is important to note that chemisorbed species, that may move on the surface, may collide with any crystal particle - the bulk particle being the closest-so that for $k=\mathrm{b}$ the distribution $f_{k}$ arising in the products of $C_{i}^{r}$ is the surface distribution $f_{\sigma}$. The activity coefficient of monatomic crystal bulk species $\mathrm{C}(\mathrm{b})$ is consistently taken to be unity in heterogeneous surface chemistry [10] and this is a fundamental difference between homogeneous and heterogeneous chemistry. The chemical operators $C_{i}, i \in S$, conserve atomic element, momentum and energy [22].

In order to simplify the reactive source term, we further observe that, at chemical equilibrium, the statistical equilibrium relation holds $\prod_{k \in \mathcal{B}^{r}} f_{k}^{\mathrm{ce}} \beta_{k \mathrm{~K}}=\prod_{j \in \mathcal{F}^{r}} f_{j}^{\mathrm{ce}} \beta_{j \mathrm{~s}}$ where the superscript ${ }^{\mathrm{ce}}$ denotes chemical equilibrium whereas 
the superscript ${ }^{\mathrm{e}}$ denotes physical equilibrium. The distributions $f_{i}^{\text {ce }}$ are given by $f_{i}^{\text {ce }}=n_{i}^{\text {ce }} \mathrm{M}_{i}$ where $n_{i}^{\text {ce }}$ denotes the chemical equilibrium value of $n_{i}$ for $i \in S$. For a collision with a bulk species, indeed with the crystal surface, we also have $f_{\sigma}^{\mathrm{e}}=n_{\sigma} \mathrm{M}_{\mathrm{b}}=f_{\sigma}^{\mathrm{ce}}$ keeping in mind that $n_{\sigma}^{\mathrm{ce}}=n_{\sigma}^{\mathrm{e}}=n_{\sigma}$ remains constant in time. At chemical equilibrium, we have equality of the chemical potentials $\sum_{j \in \mathcal{F}^{r}} \mu_{j}^{\mathrm{ce}}=\sum_{k \in \mathcal{B}^{r}} \mu_{k}^{\mathrm{ce}}$ for $r \in \mathfrak{R}$ where $\mu_{i}^{\mathrm{ce}}$ denotes the chemical potential of the $i$ th species at chemical equilibrium. The chemical potential of the $i$ th species is given by $\mu_{i}=\mu_{i}^{\text {in }}+\mathrm{w}_{i} / k_{\mathrm{B}} T_{\mathrm{w}}$ where $\mu_{i}^{\text {in }}$ is the intrinsic species chemical potential $[25,26]$. The intrinsic chemical potential is given by the usual formula $\mu_{i}^{\text {in }}=g_{i} / k_{\mathrm{B}} T=\log \left(n_{i} / \mathrm{z}_{i}\right)$ where $g_{i}$ denotes the Gibbs function of the $i$ th species, $\mathrm{z}_{i}$ the partition function per unit volume and $n_{i}$ is the local number density with the influence of the force field for $i \in S[25,26]$. For the bulk species we also have the chemical potential $\mu_{\mathrm{b}}^{\text {in }}=\log \left(n_{\sigma} / \mathrm{z}_{\mathrm{b}}\right)$ that yields an activity coefficient equal to unity in agreement with heterogeneous surface chemistry [10]. We also assume for the sake of simplicity that the formation energy of the bulk species is independent of chemisorbed species [10]. The partition functions are in the form $\mathrm{z}_{i}=\mathrm{z}_{i}^{\mathrm{tr}} \mathrm{z}_{i}^{\text {int }}$ where $\mathrm{z}_{i}^{\mathrm{tr}}$ is the translational partition function of the $i$ th species par unit volume in three dimensions and $z_{i}^{\text {int }}$ the internal energy partition function. The translational partition functions are given by $\mathrm{z}_{i}^{\mathrm{tr}}=\Lambda_{i}^{-3}$ where $\Lambda_{i}=\mathrm{h}_{\mathrm{P}} /\left(2 \pi k_{\mathrm{B}} m_{i} T_{\mathrm{w}}\right)^{1 / 2}$ denotes the thermal de Broglie wavelength of the $i$ th species and $h_{\mathrm{P}}$ the Planck constant. The stoichiometric coefficients are also constrained by the conservation of atomic elements [22]. In particular the conservation of crystal atoms in chemical reactions yields $v_{b r}+v_{s r}=0$ for $r \in \mathfrak{R}$, and the conservation of sites reads $\sum_{i \in S_{\mathrm{c}}} v_{i r}+v_{s r}=0$ for $r \in \mathfrak{R}$. Assuming then that the crystal surface remains at physical equilibrium $f_{\sigma}=f_{\sigma}^{\mathrm{e}}=n_{\sigma} \mathbf{M}_{\sigma}$, and thus at chemical equilibrium $f_{\sigma}=f_{\sigma}^{\text {ce }}$ since $n_{\sigma}$ is constant in time, dividing the integrand by the factor $\prod_{k \in \mathcal{B}^{r}} f_{k}^{\mathrm{ce}} \beta_{k \mathrm{~K}}=\prod_{j \in \mathcal{F}^{r}} f_{j}^{\mathrm{ce}} \beta_{j \mathrm{~s}}$, the source term is found after lengthy algebra in the form

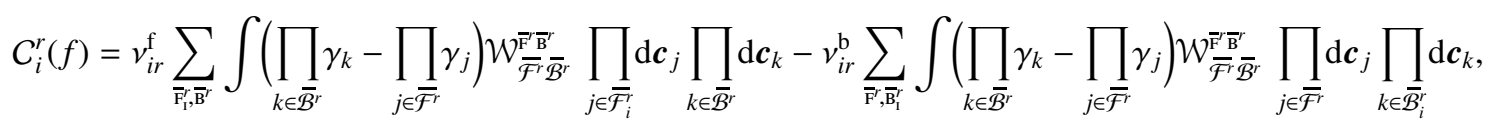

where $\gamma_{k}=f_{k} / \mathrm{m}_{k} \mathrm{z}_{k}$ for $k \in S_{\mathrm{g}} \cup S_{\mathrm{c}}$ and $\gamma_{\mathrm{s}}=(1-\theta) / \mathrm{z}_{\mathrm{s}} / \mathrm{z}_{\mathrm{b}}, 1-\theta=f_{\mathrm{s}} / f_{\sigma}$ represents the probability of a free site on the surface, $\mathcal{W}_{\overline{\mathcal{F}}^{r} \overline{\mathcal{B}}^{r}}^{\overline{\mathrm{F}}^{r}{ }^{r}}$ is an average transition probability, and we have denoted with overbars the sets $\overline{\mathcal{F}}^{r}, \overline{\mathcal{B}}^{r}, \overline{\mathrm{F}}^{r}, \overline{\mathrm{B}}^{r}$ and $\overline{\mathcal{F}}_{i}^{r}, \overline{\mathcal{B}}_{k}^{r}, \overline{\mathrm{F}}_{\mathrm{I}}^{r}$ and $\overline{\mathrm{B}}_{\mathrm{K}}^{r}$ obtained by removing any occurence of the bulk species index $\mathrm{b}$ in the corresponding set.

\section{Kinetic entropy}

The kinetic entropy per unit volume associated with the physisorbed/gas particles, the chemisorbed particles, and the coverage is defined by

$$
\mathcal{S}^{\text {kin }}=-k_{\mathrm{B}} \sum_{i \in S_{\mathrm{g}} \cup S_{\mathrm{c}} \in Q_{i}} \sum_{\mathrm{I}} \int f_{i}\left(\log \left(f_{i} / \mathrm{m}_{i} \mathrm{z}_{i}\right)-1\right) \mathrm{d} \boldsymbol{c}_{i}-k_{\mathrm{B}} \int f_{\sigma}^{\mathrm{e}}(1-\theta)\left(\log \left((1-\theta) \mathrm{Z}_{\mathrm{b}} / \mathrm{z}_{\mathrm{s}}\right)-1\right) \mathrm{d} \boldsymbol{c}_{\mathrm{s}}
$$

The resulting balance laws for $\mathcal{S}^{\text {kin }}$ is in the form $\partial_{t} \mathcal{S}^{\text {kin }}+\partial_{x} \cdot \mathcal{F}^{\text {kin }}=\mathfrak{v}^{\text {kin }}$ where $\mathcal{F}^{\text {kin }}$ is the kinetic entropy flux and $\mathfrak{v}^{\text {kin }}$ the kinetic entropy source term. The entropy source term $\mathfrak{v}^{\text {kin }}$ may be split as $\mathfrak{v}^{\text {kin }}=\mathfrak{v}_{J}+\mathfrak{v}_{\mathrm{ph}}+\mathfrak{v}_{C}$ where $\mathfrak{v}_{J}, \mathfrak{v}_{\mathrm{ph}}$, and $\mathfrak{v}_{C}$ are respectively due to inert collisions between gas/physisorbed species, collision between phonons and particles and reactive collisions, and all these contributions $\mathfrak{v}_{J}, \mathfrak{v}_{\mathrm{ph}}$, and $\mathfrak{v}_{C}$ are shown to be nonnegative.

\section{MULTISCALE FRAMEWORK}

\section{Fluid scaling}

Let $T^{\star}$ denotes a characteristic temperature, $n^{\star}$ a number density, $\tau_{\mathrm{p}}^{\star}$ a collision time, $m$ a particle mass, $c^{\star}=$ $\left(k_{\mathrm{B}} T^{\star} / m\right)^{1 / 2}$ a characteristic thermal velocity, $f^{\star}=n^{\star} / c^{\star 3}$ a characteristic particle distribution and $\lambda^{\star}=\tau_{\mathrm{p}}^{\star} c^{\star}$ the mean free path. Similarly, let $\tau_{\mathrm{f}}^{\star}$ denotes a characteristic fluid time, $l^{\star}=\tau_{\mathrm{f}}^{\star} c^{\star}$ the fluid length, $\tau_{\mathrm{ph}}^{\star}$ a characteristic time for phonon interaction related to characteristic transition probabilities $1 / \tau_{\mathrm{ph}}^{\star}=\mathrm{W}_{i, \mathrm{ph}}^{\star} c^{\star 3}$, and $\delta^{\star}$ a typical length characteristic of the range of surface potential. Dividing the kinetic equation (2) by $n^{\star} / \tau_{\mathrm{f}}^{\star} c^{\star 3}$, the resulting rescaled equations involve the dimensionless parameters $\epsilon_{\mathrm{p}}=\tau_{\mathrm{p}}^{\star} / \tau_{\mathrm{f}}^{\star}=\lambda^{\star} / l^{\star}, \epsilon_{\mathrm{ph}}=\tau_{\mathrm{ph}}^{\star} / \tau_{\mathrm{f}}^{\star}$, and $\epsilon=\delta^{\star} / l^{\star}$. The characteristic times and lengths at the solid/gas interface are generally such that $\tau_{\mathrm{ph}}^{\star} \leq \tau_{\mathrm{p}}^{\star} \ll \tau_{\mathrm{f}}^{\star}$ and $\delta^{\star} \leq \lambda^{\star} \ll l^{\star}$ and $\epsilon_{\mathrm{p}}$ represents the Knudsen number [12]. Since our aim in this work is to derive fluid boundary conditions, it is assumed that the 
small parameters $\epsilon_{\mathrm{p}}, \epsilon_{\mathrm{ph}}$, and $\epsilon$ are of the same asymptotic order of magnitude $\epsilon_{\mathrm{ph}}=\alpha_{\mathrm{ph}} \epsilon$ and $\epsilon_{\mathrm{p}}=\alpha_{\mathrm{p}} \epsilon$ where $\alpha_{\mathrm{ph}}$ and $\alpha_{\mathrm{p}}$ are positive constants. From a physical point of view, this means that particle collisions and phonon interactions are considered to be fast in comparison with fluid time and that both the mean free path $\lambda^{\star}$ and the surface potential characteristic range $\delta^{\star}$ are considered to be small in comparison with the fluid length $l^{\star}$. This scaling may be seen as the simplest fluid scaling of the adsorbate layer model.

Keeping the same notation for rescaled quantities, the resulting rescaled kinetic equations are in the form

$$
\partial_{t} f_{i}+\boldsymbol{c}_{i \|} \cdot \boldsymbol{\partial}_{\|} f_{i}+c_{i z} \partial_{z} f_{i}-\frac{1}{m_{i}} \partial_{z} \mathrm{w}_{i} \partial_{c_{i z}} f_{i}=\frac{1}{\alpha_{\kappa} \epsilon} \sum_{j \in S_{\mathrm{g}}} J_{i j}\left(f_{i}, f_{j}\right)+\frac{1}{\alpha_{\mathrm{ph}} \epsilon} J_{i, \mathrm{ph}}\left(f_{i}\right)+C_{i}, \quad i \in S,
$$

where $\boldsymbol{c}_{i \|}$ and $c_{i z}$ denote the tangential and normal velocities with $\boldsymbol{c}_{i}=\left(\boldsymbol{c}_{i \|}, c_{i z}\right)^{t}$. The chemistry operators $C_{i}, i \in S$, are therefore assumed to be slow in comparison with the inert collision operators $J_{i j}, i, j \in S_{\mathrm{g}}$, and $J_{i, \mathrm{ph}}, i \in S$.

\section{Expansion in the gas}

Far from the surface, the rescaled equations coincide with the traditional scaling of the Chapman-Enskog method using the formal expansion parameter $\alpha_{\kappa} \epsilon$. The standard Enskog expansions of the species distributions are also modified since the fluid convection velocity $\boldsymbol{v}_{\mathrm{g}}$ near the surface $z=0$ is of first order in $\epsilon$. The corrected expansion of the distribution function $f_{\mathrm{g} i}$ valid in the gas phase near the surface is denoted in the form $f_{\mathrm{g} i}=f_{\mathrm{g} i}^{(0)}+\epsilon f_{\mathrm{g} i}^{(1)}+O\left(\epsilon^{3}\right)$ and after some algebra, it is obtained that

$$
f_{\mathrm{g} i}^{(0)}=n_{\mathrm{g} i}\left(\frac{m_{i}}{2 \pi k_{\mathrm{B}} T_{\mathrm{w}}}\right)^{3 / 2} \frac{a_{i \mathrm{I}}}{\mathrm{z}_{i}^{\text {int }}} \exp \left(-\frac{m_{i}\left|c_{i}\right|^{2}}{2 k_{\mathrm{B}} T_{\mathrm{w}}}-\frac{\mathrm{E}_{i \mathrm{I}}}{k_{\mathrm{B}} T_{\mathrm{w}}}\right), \quad f_{\mathrm{g} i}^{(1)}=\left(\frac{m_{i} c_{i z} \bar{v}_{\mathrm{g} z}}{k_{\mathrm{B}} T_{\mathrm{g}}}+\alpha_{\kappa} \phi_{\mathrm{g} i}^{(1)}\right) f_{\mathrm{g} i}^{(0)},
$$

where $f_{\mathrm{g} i}^{(0)}$ is the zero velocity Maxwellian, $n_{\mathrm{g} i}\left(t, \boldsymbol{x}_{\mathrm{n}}, z\right)$ the number density, $\phi_{\mathrm{g} i}^{(1)}$ the usual first-order perturbation in the Navier-Stokes regime with a zero mass average velocity, and $\bar{v}_{\mathrm{g} z}$ the rescaled normal gas velocity with $\boldsymbol{v}_{\mathrm{g}}=\epsilon \bar{v}_{\mathrm{g} z} \boldsymbol{e}_{z}$.

\section{Multiscale expansion}

Since the surface potentials $\mathrm{w}_{i}, i \in S$, depend on the layer coordinate $\zeta=z / \epsilon$, the problem appears as multiscale since it involves both $z$ as well as $\zeta=z / \epsilon$. The physisorbed/gas distributions $f_{i}, i \in S_{\mathrm{g}}$, are expanded in the multiscale form

$$
f_{i}=\sum_{j} \epsilon^{j} f_{\mathrm{g} i}^{(j)}\left(t, \boldsymbol{x}_{\mathrm{\|}}, z, \boldsymbol{c}_{i}\right)+\sum_{j} \epsilon_{\mathrm{lc} i}^{j} \mathrm{f}_{\mathrm{l}}^{(j)}\left(t, \boldsymbol{x}_{\mathrm{\|}}, \zeta, \boldsymbol{c}_{i}\right), \quad i \in S_{\mathrm{g}},
$$

where $\sum_{j} \epsilon^{j} f_{\mathrm{g} i}^{(j)}\left(t, \boldsymbol{x}_{\mathrm{l}}, z, \boldsymbol{c}_{i}\right)$ is the outer gas expansion and $\sum_{j} \epsilon^{j} \mathrm{f}_{\mathrm{lc} i}^{(j)}\left(t, \boldsymbol{x}_{\mathrm{l}}, \zeta, \boldsymbol{c}_{i}\right)$ the adsorbate layer corrector expansion. The adsorbate layer correctors $\mathrm{f}_{\mathrm{lci}}^{(j)}\left(t, \boldsymbol{x}_{\|}, \zeta, \boldsymbol{c}_{i}\right), j \geq 0$, have to converge to zero as $\zeta \rightarrow \infty$ in such a way that the gas expansion of $f_{\mathrm{g} i}$ is recovered in the gas phase.

The inner expansion of the distribution $\mathrm{f}_{i}, i \in S_{\mathrm{g}}$, in the physisorbate is next obtained by first expanding the outer expansion around $z=0$ with $z=\epsilon \zeta$ and adding the corrector expansion. The inner expansion $\mathrm{f}_{\mathrm{g} i}$ of $f_{\mathrm{g} i}$ is written in the form $\mathrm{f}_{\mathrm{g} i}\left(t, \boldsymbol{x}_{\|}, \zeta, \boldsymbol{c}_{i}\right)=f_{\mathrm{g} i}\left(t, \boldsymbol{x}_{\|}, \epsilon \zeta, \boldsymbol{c}_{i}\right)=\sum_{j} \epsilon_{\mathrm{g} i}^{j}\left(t, \boldsymbol{x}_{\mathrm{\|}}, \zeta, \boldsymbol{c}_{i}\right)$ and it is found with $z=\epsilon \zeta$ that $\mathrm{f}_{\mathrm{g} i}^{(0)}\left(t, \boldsymbol{x}_{\mathrm{\|}}, \zeta, \boldsymbol{c}_{i}\right)=f_{\mathrm{g} i}^{(0)}\left(t, \boldsymbol{x}_{\mathrm{\|}}, 0, \boldsymbol{c}_{i}\right)$ and $\mathrm{f}_{\mathrm{g} i}^{(1)}=f_{\mathrm{g} i}^{(1)}\left(t, \boldsymbol{x}_{\mathrm{\|}}, 0, \boldsymbol{c}_{i}\right)+\zeta \partial_{z} f_{\mathrm{g} i}^{(0)}\left(t, \boldsymbol{x}_{\mathrm{\|}}, 0, \boldsymbol{c}_{i}\right)$. The inner expansion of the distribution $f_{i}$ in the physisorbate is denoted by $\mathrm{f}_{i}=\sum_{j} \epsilon_{i}^{j} \mathrm{f}_{i}^{(j)}\left(t, \boldsymbol{x}_{\mathrm{ll}}, \zeta, \boldsymbol{c}_{i}\right)$ and its coefficients are thus $\mathrm{f}_{i}^{(j)}=\mathrm{f}_{\mathrm{g} i}^{(j)}+\mathrm{f}_{\mathrm{lci}}^{(j)}$ for $j \geq 0$ with a polynomial $\mathrm{f}_{\mathrm{g} i}^{(j)}$ in terms of $\zeta$ arising from the Taylor expansion of $f_{\mathrm{g} i}$ and a thin layer corrector $\mathrm{f}_{\mathrm{lc} i}^{(j)}$. The chemisorbate, free site, bulk and surface distributions are expanded in the simpler form

$$
f_{i}=\sum_{j} \epsilon^{j} \mathrm{f}_{i}^{(j)}\left(t, \boldsymbol{x}_{\|}, \zeta, \boldsymbol{c}_{i}\right), \quad i \in S_{\mathrm{c}} \cup\{\mathrm{s}, \mathrm{b}\},
$$

since such distributions are localized in the adsorption layer and only involve the inner layer coordinate $\zeta$. Since $\zeta$ is the proper normal coordinate of the adsorbate layer, the rescaled Boltzmann equations are in the form

$$
\partial_{t} f_{i}+\boldsymbol{c}_{i \|} \cdot \boldsymbol{\partial}_{\|} f_{i}+\frac{1}{\epsilon} c_{i z} \partial_{\zeta} f_{i}-\frac{1}{\epsilon} \frac{1}{m_{i}} \partial_{\zeta} \mathrm{w}_{i} \partial_{c_{i z}} f_{i}=\frac{1}{\alpha_{\kappa} \epsilon} \sum_{j \in S_{\mathrm{g}}} J_{i j}\left(f_{i}, f_{j}\right)+\frac{1}{\alpha_{\mathrm{ph}} \epsilon} J_{i, \mathrm{ph}}\left(f_{i}\right)+C_{i}, \quad i \in S,
$$

where $\partial_{\text {II }}$ denotes the tangential spatial derivative operator. A similar rescaled equation is also obtained for the inner distributions $\mathrm{f}_{\mathrm{g} i}, i \in S_{\mathrm{g}}$, of the gas distributions without the potential, phonon and source terms. 


\section{ASYMPTOTIC ANALYSIS OF THE ADSORBATE}

\section{Zeroth order expansion in the physisorbate}

In the physisorbate, using (13), the $i$ th species equation is found to be

$$
c_{i z} \partial_{\zeta} \mathrm{f}_{i}^{(0)}-\frac{1}{m_{i}} \partial_{\zeta} \mathrm{w}_{i} \partial_{c_{i z}} \mathrm{f}_{i}^{(0)}=\frac{1}{\alpha_{\kappa}} \sum_{j \in S_{\mathrm{g}}} J_{i j}\left(\mathrm{f}_{i}^{(0)}, \mathrm{f}_{j}^{(0)}\right)+\frac{1}{\alpha_{\mathrm{ph}}} J_{i, \mathrm{ph}}\left(\mathrm{f}_{i}^{(0)}\right), \quad i \in S_{\mathrm{g}} .
$$

We multiply (14) by $\log \left(\mathrm{f}_{i}^{(0)} / \mathrm{m}_{i}\right)$, make use of $\partial_{\mathcal{A}}(\mathcal{A}(\log \mathcal{A}-1))=\log \mathcal{A}$, use the properties of the modified Maxwellian $\mathrm{m}_{i}$, and then integrate over $\zeta \in(0, \infty), \boldsymbol{c}_{i} \in \mathbb{R}^{3}$, and sum over $\mathrm{I} \in \mathcal{Q}_{i}$ and $i \in S_{\mathrm{g}}$. Using that $\mathrm{f}_{i}^{(0)} \rightarrow 0$ as $\zeta \rightarrow 0$ since the surface repulsive potential repel all physisorbed/gas particles, and $\mathrm{f}_{i}^{(0)}\left(t, \boldsymbol{x}_{\mathrm{\|}}, \zeta, \boldsymbol{c}_{i}\right) \rightarrow f_{\mathrm{g} i}^{(0)}\left(t, \boldsymbol{x}_{\|}, 0, \boldsymbol{c}_{i}\right)$ as $\zeta \rightarrow \infty$ since the corrector $\mathrm{f}_{\mathrm{lc} i}^{(0)}$ goes to zero as $\zeta \rightarrow \infty$, it is established that entropy production in the physisorbate vanish. Therefore $\mathrm{f}_{i}^{(0)}$ is a Maxwellian with temperature $T_{\mathrm{w}}$ and zero average velocity since $J_{i, \mathrm{ph}}\left(\mathrm{f}_{i}^{(0)}\right)=0$. Writting this Maxwellian for convenience as $\mathrm{f}_{i}^{(0)}=\bar{n}_{i} \exp \left(-\frac{\mathrm{w}_{i}}{k_{\mathrm{B}} T_{\mathrm{w}}}\right) \mathrm{M}_{i}$ and substituting this identity in (14), it is obtained that $\partial_{\zeta} \bar{n}_{i}=0$ so that $\bar{n}_{i}$ is independent of $\zeta$. The constant $\bar{n}_{i}$ is identified by letting $\zeta \rightarrow \infty$ and it is obtained that $\bar{n}_{i}=n_{\mathrm{g} i}\left(t, x_{\|}, 0\right)$, where $n_{\mathrm{g} i}\left(t, \boldsymbol{x}_{\mathrm{II}}, z\right)=\sum_{\mathrm{I} \in \mathcal{Q}_{i}} \int f_{\mathrm{g} i}^{(0)} \mathrm{d} \boldsymbol{c}_{i}$ is the gas number density, so that

$$
\mathrm{f}_{i}^{(0)}=n_{i} \mathrm{M}_{i}, \quad n_{i}\left(t, \boldsymbol{x}_{\mathrm{\|}}, \zeta\right)=n_{\mathrm{g} i}\left(t, \boldsymbol{x}_{\mathrm{\|}}, 0\right) \exp \left(-\frac{\mathrm{w}_{i}(\zeta)}{k_{\mathrm{B}} T_{\mathrm{w}}}\right), \quad i \in S_{\mathrm{g}}
$$

This expression of $n_{i}\left(t, x_{\mathrm{ll}}, \zeta\right)$ establishes that the $i$ th species is naturally distributed like $\exp \left(-\mathrm{w}_{i}(\zeta) / k_{\mathrm{B}} T_{\mathrm{w}}\right)$ as was expected based of physical grounds $[12,25]$ and that the $i$ th species in the physisorbate is at equilibrium with the bath of gas of the $i$ th species with number density $n_{\mathrm{g} i}\left(t, \boldsymbol{x}_{\|}, 0\right)$ arising from the gas phase.

\section{Zeroth order expansion in the chemisorbate}

Proceeding as for the physisorbate, it is established that for the chemisorbed and the crystal species

$$
f_{i}=n_{i} \mathrm{M}_{i}, \quad n_{i}\left(t, \boldsymbol{x}_{\mathrm{II}}, \zeta\right)=\bar{n}_{i}\left(t, \boldsymbol{x}_{\mathrm{\| I}}\right) \exp \left(-\frac{\mathrm{w}_{i}(\zeta)}{k_{\mathrm{B}} T_{\mathrm{w}}}\right) \quad i \in S_{\mathrm{c}} \cup\{\mathrm{s}, \mathrm{b}\},
$$

where $\bar{n}_{i}\left(t, x_{11}\right)$ only depends on time and tangential coordinate. We may also introduce the number of chemisorbed particles per unit surface $\widetilde{n}_{i}\left(t, x_{\mathrm{\| I}}\right)=\int n_{i} \mathrm{~d} z$ related to $\bar{n}_{i}$ via a configuration integral $\widetilde{n}_{i}=\bar{n}_{i} \int \exp \left(-\frac{\mathrm{w}_{i}}{k_{\mathrm{B}} T_{\mathrm{w}}}\right) \mathrm{d} z$ for $i \in S_{\mathrm{c}} \cup\{\mathrm{s}, \mathrm{b}\}$. A similar structure holds for the standard equilibrium surface distribution $f_{\sigma}^{\mathrm{e}}=\mathrm{f}_{\sigma}=\mathrm{f}_{\sigma}^{(0)}$ with a number density of the surface layer $n_{\sigma}$ in the form $n_{\sigma}=\bar{n}_{\sigma} \exp \left(-\frac{\mathrm{w}_{\sigma}}{k_{\mathrm{B}} T_{\mathrm{w}}}\right)$. The quantities $\bar{n}_{\sigma}$ and $\widetilde{n}_{\sigma}$ are characteristic of the crystal surface and its orientation. From the structure of the free site distribution $f_{\mathrm{s}}^{(0)}$ and that of the crystal layer standard distribution $f_{\sigma}^{\mathrm{e}}=\mathrm{f}_{\sigma}^{(0)}$ we deduce that at zeroth order the probability of free site $1-\bar{\theta}=\mathrm{f}_{\mathrm{s}}^{(0)} / \mathrm{f}_{\sigma}^{(0)}$ simplifies into $1-\bar{\theta}=\bar{n}_{\mathrm{s}} / \bar{n}_{\sigma}$ and therefore only depends on time and tangential coordinate $\bar{\theta}=\bar{\theta}\left(t, x_{11}\right)$. This property was assumed to hold in previous work but is established here from the kinetic equations. Introducing the number of free site per unit surface $\widetilde{n}_{\mathrm{s}}=\int n_{\mathrm{s}} \mathrm{d} z$ we also have $\widetilde{n}_{\mathrm{s}}=(1-\bar{\theta}) \widetilde{n}_{\sigma}$. Since the chemisorbate is assumed to be monolayer we have the natural constraints $\sum_{i \in S_{\mathrm{c}}} \widetilde{n}_{i}+\widetilde{n}_{\mathrm{s}}=\widetilde{n}_{\sigma}$ and $\widetilde{n}_{\mathrm{b}}=\sum_{i \in S_{\mathrm{c}}} \widetilde{n}_{i}$

\section{Species fluid boundary condition}

In the physisorbate, subtracting the gas inner equation from (13), integrating with respect to the velocity variable, summing over $\mathrm{I} \in \mathcal{Q}_{i}$, and keeping only zeroth order terms, it is obtained that

$$
\partial_{t} \sum_{\mathrm{I} \in \mathcal{Q}_{i}} \int\left(\mathrm{f}_{i}^{(0)}-\mathrm{f}_{\mathrm{g} i}^{(0)}\right) \mathrm{d} \boldsymbol{c}_{i}+\partial_{\zeta} \sum_{\mathrm{I} \in \mathcal{Q}_{i}} \int c_{i z}\left(\mathrm{f}_{i}^{(1)}-\mathrm{f}_{\mathrm{g} i}^{(1)}\right) \mathrm{d} \boldsymbol{c}_{i}=\sum_{\mathrm{r} \in \mathcal{Q}_{i}} \int C_{i}^{(0)} \mathrm{d} \boldsymbol{c}_{i},
$$

where $C_{i}^{(0)}$ denotes the chemical production terms evaluated with the Maxwellian distributions $\mathrm{f}_{i}^{(0)}, i \in S$. Moreover, we have the estimate $\partial_{t} n_{\mathrm{g} i}\left(t, x_{\mathrm{n}}, 0\right)=O(\epsilon)$ since $v_{\mathrm{g}}=O(\epsilon)$ so that $\partial_{t}\left(\mathrm{f}_{i}^{(0)}-\mathrm{f}_{\mathrm{g} i}^{(0)}\right)=O(\epsilon)$ and the first term vanishes at zeroth 
order. Integrating with respect to $\zeta$ over $\zeta \in(0, \infty)$, using that $\mathrm{f}_{i}^{(1)}-\mathrm{f}_{\mathrm{g} i}^{(1)}=\mathrm{f}_{\mathrm{lci}}^{(1)}$ goes to zero as $\zeta \rightarrow \infty$, that $\mathrm{f}_{i}^{(1)}$ goes to zero as $\zeta \rightarrow 0$, since there are not anymore particles when $\zeta \rightarrow 0$, that $\mathrm{f}_{\mathrm{g} i}^{(1)}\left(t, \boldsymbol{x}_{\|}, \zeta, \boldsymbol{c}_{i}\right)=f_{\mathrm{g} i}^{(1)}\left(t, \boldsymbol{x}_{\|}, 0, \boldsymbol{c}_{i}\right)+\zeta \partial_{z} f_{\mathrm{g} i}^{(0)}\left(t, \boldsymbol{x}_{\|}, 0, \boldsymbol{c}_{i}\right)$, and $\zeta \partial_{z} f_{\mathrm{g} i}^{(0)}$ go to zero as $\zeta \rightarrow 0$, we obtain $\sum_{\mathrm{1} \in Q_{i}} \int c_{i z} f_{\mathrm{g} i}^{(1)}\left(t, \boldsymbol{x}_{\mathrm{n}}, 0, \boldsymbol{c}_{i}\right) \mathrm{d} \boldsymbol{c}_{i}=\sum_{\mathrm{1} \in Q_{i}} \int C_{i}^{(0)} \mathrm{d} \boldsymbol{c}_{i} \mathrm{~d} \zeta$. The flux of particles of the $i$ th species in the gas may be evaluated in terms of the Stefan velocity and the diffusive flux $\mathcal{F}_{i}\left(t, \boldsymbol{x}_{\|}, 0\right)$ of the $i$ th species and we have obtained that the boundary condition for the $i$ th species reads

$$
n_{\mathrm{g} i}\left(t, \boldsymbol{x}_{\|}, 0\right) v_{\mathrm{g} z}\left(t, \boldsymbol{x}_{\|}, 0\right)+\mathcal{F}_{i}\left(t, \boldsymbol{x}_{\|}, 0\right)=\mathfrak{w}_{i}^{0}, \quad \mathfrak{w}_{i}^{0}=\sum_{\mathrm{i} \in Q_{i}} \int C_{i}^{(0)} \mathrm{d} \boldsymbol{c}_{i} \mathrm{~d} z, \quad i \in S_{\mathrm{g}},
$$

where $\mathfrak{w}_{i}^{0}$ denotes for short the zeroth order number of particles of the $i$ th species produced by unit volume and unit time by surface chemistry in the adsorbate.

On the other hand, the integrated mass conservation equation for the chemisorbed and crystal species may be obtained with a similar procedure and yields that

$$
\partial_{t} \widetilde{n}_{\mathrm{c} i}\left(t, \boldsymbol{x}_{\|}\right)=\mathfrak{w}_{i}^{0}, \quad \mathfrak{w}_{i}^{0}=\sum_{\mathrm{i} \in Q_{i}} \int C_{i}^{(0)} \mathrm{d} \boldsymbol{c}_{i} \mathrm{~d} z, \quad i \in S_{\mathrm{c}} \cup\{\mathrm{s}, \mathrm{b}\},
$$

where $\widetilde{n}_{\mathrm{c} i}\left(t, \boldsymbol{x}_{\|}\right)=\bar{n}_{\mathrm{c} i}\left(t, \boldsymbol{x}_{\|}\right) \int \exp \left(-\mathrm{w}_{i} / k_{\mathrm{B}} T_{\mathrm{w}}\right) \mathrm{d} z$ represents the total amount of chemisorbed or crystal species available in the layer per unit surface and may be interpreted as the natural surface number density. We also have natural relations obtained from the monolayer structure of the chemisorbate $\widetilde{n}_{\mathrm{b}}=\sum_{i \in S_{\mathrm{c}}} \widetilde{n}_{i}$ and $\sum_{i \in S_{\mathrm{c}}} \widetilde{n}_{i}=\bar{\theta} \widetilde{n}_{\sigma}$ and $\widetilde{n}_{\mathrm{s}}=$ $(1-\bar{\theta}) \widetilde{n}_{\sigma}$. From the conservation of sites and of $\mathcal{C}$ atoms we have $\mathfrak{w}_{\mathrm{s}}^{0}+\mathfrak{w}_{\mathrm{b}}^{0}=0$ and $\mathfrak{w}_{\mathrm{b}}^{0}=\sum_{i \in S_{\mathrm{c}}} \mathfrak{w}_{i}^{0}$. We have therefore recovered the traditional mass fluid boundary conditions at a surface with adsorption including the equation for the gas, the chemisorbed species and the coverage [10,22].

\section{Surface chemical reaction}

The integrated surface chemistry term $\int C_{i}^{(0)} \mathrm{d} c_{i} \mathrm{~d} z$ evaluated with the distributions $\mathrm{f}_{i}^{(0)}, i \in S$, is obtained in the form

$$
\mathfrak{w}_{i}^{0}=\sum_{\mathrm{r} \in Q_{i}} \int C_{i}^{(0)} \mathrm{d} \boldsymbol{c}_{i} \mathrm{~d} z=\sum_{r \in \Re}\left(v_{i r}^{\mathrm{f}}-v_{i r}^{\mathrm{b}}\right) \chi_{r},
$$

where $\chi_{r}$ denotes the rate of progress of the $r$ th reaction. This rate is found in the form

$$
\chi_{r}=\mathcal{K}_{r}\left(\prod_{k \in \mathcal{F}^{r} \cap S_{\mathrm{g}}} \frac{\widetilde{n}_{\mathrm{g} k}}{\mathrm{z}_{k}} \prod_{k \in \mathcal{F} r \cap S_{\mathrm{c}}} \frac{\bar{n}_{k}}{\mathrm{z}_{k}} \prod_{\mathcal{F}^{r} \cap\{\mathrm{s}\}} \frac{1-\bar{\theta}}{\mathrm{z}_{\mathrm{s}} / \mathrm{z}_{\mathrm{b}}}-\prod_{k \in \mathcal{G}^{r} \cap S_{\mathrm{g}}} \frac{\widetilde{n}_{\mathrm{g} k}}{\mathrm{z}_{k}} \prod_{k \in \mathcal{B}^{r} \cap S_{\mathrm{c}}} \frac{\bar{n}_{k}}{\mathrm{z}_{k}} \prod_{\mathcal{B}^{r} \cap\{\mathrm{s}\}} \frac{1-\bar{\theta}}{\mathrm{z}_{\mathrm{s}} / \mathrm{z}_{\mathrm{b}}}\right),
$$

where $\widetilde{n}_{\mathrm{g} k}$ is a shorter notation for $\widetilde{n}_{\mathrm{g} k}=n_{\mathrm{g} k}\left(t, \boldsymbol{x}_{\mathrm{n}}, 0\right)$ and $\mathcal{K}_{r}$ is the reaction constant of the $r$ th reaction

$$
\mathcal{K}_{r}=\sum_{\mathrm{F}^{r}, \mathrm{~B}^{r}} \int \mathcal{W}_{\mathcal{F}^{r} \mathcal{B}^{r}}^{\mathrm{F}^{r} \mathrm{~B}^{r}} \prod_{j \in \mathcal{F}^{r}} \mathrm{~d} \boldsymbol{c}_{j} \prod_{k \in \mathcal{B}^{r}} \mathrm{~d} \boldsymbol{c}_{k}
$$

Using notably $\bar{n}_{i} / \mathrm{z}_{i}=\widetilde{n}_{i} / \widetilde{\mathrm{z}}_{i}$ for $i \in S_{\mathrm{c}}$, where $\widetilde{\mathrm{z}}_{i}$ denotes the partition function of the chemisorbed surface species with a configuration integral [21] $\widetilde{\mathrm{z}}_{i}=\mathrm{z}_{i} \int \exp \left(-\frac{\mathrm{w}_{i}}{k_{\mathrm{B}} T_{\mathrm{w}}}\right) \mathrm{d} z$, we may also write the rate of progress of the $r$ th reaction as

$$
\chi_{r}=\mathcal{K}_{r}\left(\prod_{k \in S_{\mathrm{g}}} \widetilde{n}_{\mathrm{g} k}^{r_{k r}^{\mathrm{f}}} \prod_{k \in S_{\mathrm{c}}} \widetilde{n}_{k}^{r_{k r}^{\mathrm{f}}}(1-\bar{\theta})^{v_{\mathrm{sr}}^{\mathrm{f}}}-\frac{1}{\mathcal{K}_{r}^{\mathrm{ce}}} \prod_{k \in S_{\mathrm{g}}} \widetilde{n}_{\mathrm{g} k}^{v_{k r}^{\mathrm{b}}} \prod_{k \in S_{\mathrm{c}}} \widetilde{n}_{k}^{v_{k r}^{\mathrm{b}}}(1-\bar{\theta})^{v_{\mathrm{sr}}^{\mathrm{b}}}\right),
$$

where $\mathcal{K}_{r}^{\mathrm{ce}}$ denotes the equilibrium constant of the $r$ th reaction. These expresssions are in particular compatible with the atomic site formalism as well as with the open site formalism traditionally used for the description of complex heterogeneous surface mechanisms [3, 10]. For a simple adsorption reaction, the Langmuir isotherm is recovered at surface chemical equilibrium [18]. The saturation factor $1-\bar{\theta}$ is traditionally integrated into chemical potential of the chemisorbed species but it still originates from the surface crystal species $\mathcal{C}(s)$. 


\section{CONCLUSION}

A kinetic model describing adsorption or desorption of gas particles on a crystal surface as well as surface chemical reactions has been introduced, at a scale intermediate between molecular and macroscopic. The phonon and surface collision terms have simplified by assuming that the phonons are at equilibrium and that the surface reactions are slow. The coupled system of gas/physisorbate and chemisorbate kinetic equations satisfies the $\mathrm{H}$ theorem. Using a fluid scaling and a multiscale framework, the structure of the adsorbate layer has been analyzed in terms of interaction potentials and the traditional fluid species boundary conditions have been recovered.

Adding phonon equations would be of high scientific interest as well as considering nonisothermal systems or investigating first order accurate expansions with surface diffusion. Considering the periodic dependence of the potentials with respect to the tangential coordinates would also be of high scientific interest. Multitemperature flows as well as state to state models also involve gas surface interactions of paramount importance for reentry and may also be investigated with similar models. Last but not least, numerical simulations could be used to validate the modeling framework as well as the Chapman-Enskog expansions.

\section{ACKNOWLEDGMENTS}

The first author would like to acknowledge the generous support of National Center for Theoretical Sciences, National Taiwan University and of the Department of Mathematics, National Cheng Kung University. The present work was initiated while the second author was staying at the Department of Mechanical Engineering and Science, Kyoto University as a JSPS short-term fellow and developped while invited by by the National Center for Theoretical Sciences (NCTS) of National Taiwan University.

\section{REFERENCES}

[1] D. Bruno, M. Cacciatore, S. Longo, and M. Rutigliano, Chem. Phys. Letters 320, 245-254 (2000).

[2] E. Kustova, E. Nagnibeda, I. Armenise, and M. Capitelli, J. Thermo. Heat Transfer 16, 238-244 (2002).

[3] O. Deutschmann, U. Riedel, and J. Warnatz, ASME. J. Heat Transfer 117, 495-501 (1995).

[4] V. Giovangigli and S. Candel, Comb. Sci. and Tech. 48, 1-30 (1986).

[5] N. Meynet, A. Bentaïb, and V. Giovangigli, Combust. Flame 161, 2192-2202 (2014).

[6] Y. Sone, Molecular Gas Dynamics, Theory, Techniques, and Applications, Birkhaüser, Boston (2007).

[7] K. Aoki, Y. Sone, and T. Yamada, Phys. Fluids A 2, 1867-1878 (1990).

[8] A. Ern, V. Giovangigli, and M. Smooke, J. Comp. Phys. 126, 21-39 (1996).

[9] A. Groß, Theoretical Surface Science, A microscopic Perspective, Springer Verlag, Berlin (2009).

[10] R.J. Kee, M.E. Coltrin, and P. Glarborg, Chemically Reacting Flow, Wiley, Hoboken (2003).

[11] V.D. Borman, S.Yu. Krylov, A.V. Prosyanov, and A.M. Kharitonov, Sov. Phys. JETP 63, 43-56 (1986).

[12] V.D. Borman, S.Yu. Krylov, and A.V. Prosyanov, Sov. Phys. JETP 67, 2110-2121 (1988).

[13] A.V. Bogdanov, G.V. Dubrovskiy, M.P. Krutikov, D.V. Kulginov, and V.M. Strelchenya, Interaction of gases with surfaces, Springer Verlag, Berlin 1995.

[14] A. Yu. Pankov, V.D. Borman, and S.Yu. Krylov,, J. Exp. Theor. Phys. 86, 737-744 (1998).

[15] A. Frezzotti and L. Gibelli, Proc. IMechE, J. Mech. Eng. Science 222, 787-795 (2008).

[16] K. Aoki, P. Charrier, and P. Degond, Kin. Rel. Models 4, 53-85 (2011).

[17] S. Brull, P. Charrier, and L. Mieussens, Kin. Rel. Models 7, 219-251 (2014).

[18] K. Aoki, V. Giovangigli, and M. Hattori, AIP Conference Proceedings 1786, 100005 (2016).

[19] E.M. Lifshitz and L.P. Pitaevskii, Physical Kinetics, Landau and Lifshitz course on theoretical physics, Volume 10, Elsevier, (1981).

[20] A. Rossani, Physica A 305, 323-329 (2002).

[21] D.M. Ruthven, Principles of Adsorption \& Adsorption Processes, John Wiley \& Sons, New York, (1984).

[22] V. Giovangigli, Multicomponent Flow Modeling, Birkhaüser, Boston (1999).

[23] E. Nagnibeda and E. Kustova, Non-Equilibrium Reacting Gas Flow, Springer Verlag, Berlin (2009).

[24] N.N. Bogoliubov and N.N. Bogoliubov (Jr), Teoret. Mat. Fiz. 43, 3-17 (1980).

[25] J.S. Rowlinson and B. Widom, Molecular Theory of Capillarity, Dover publications, Mineola, 2002.

[26] J.G. Powles, S.E. Baker, and W.A.B. Evans, J. Chem. Phys. 101, 4098-4102 (1994). 